\title{
Comparative Efficacy of Advanced Surgical Hemostats in Canine Hepatotomy
}

\author{
Syed Wasim Abbass,", Hamid Akbar ${ }^{1}$, Muhammad Arif Khan ${ }^{1}$, Muhammad NaveedAli ${ }^{1}$, \\ Ghulam Abbas', Muhammad Asif ${ }^{1}$ \\ ${ }^{1}$ Department of Clinical Medicine and Surgery, University of Veterinary and Animal Sciences, Pakistan
${ }^{2}$ Department of Animal Production, Riphah College of Veterinary Sciences, Riphah International University, Pakistan
}

Copyright $\bigcirc 2018$ by authors, all rights reserved. Authors agree that this article remains permanently open access under the terms of the Creative Commons Attribution License 4.0 International License

\begin{abstract}
In the present study Hemostatic effects of Surgicel Snow and Surgicel Fibrillar in 12 cases in dogs were observed. The dogs were divided into three groups via group $\mathrm{A}, \mathrm{B}$ and $\mathrm{C}$ with four dogs in each group. All three groups were anesthetized by the combination of xylazine $(1.1 \mathrm{mg} / \mathrm{kg})$ and ketamine $(5.5 \mathrm{mg} / \mathrm{kg})$ administered intravenous. Group A received Surgical Gauze, Surgicel Snow was employed in Group B and Surgicel Fibrillar was used in Group C. Hemostasis time, Liver function test (LFT), Complete blood count (CBC), Temperature, Pulse rate and Respiratory rate (TPR), Ultrasonography were evaluated. Hemostasis time was significantly less in animals who had received a Surgicel Snow compared to Surgicel Fibrillar and Surgical Gauze. LFT values were significantly different among all three groups. CBC values also differ among all groups. In TPR there were no significant differences in all groups. All groups showed no changes during Ultrasonography of liver. Surgicel Snow is better hemostat than Surgicel Fibrillar with minimum blood loss and least effects on liver function.
\end{abstract}

Keywords Surgicel Snow, Surgicel Fibrillar, Surgical Gauze, Bleeding, Liver, Dog

\section{Introduction}

Hepatic surgeries are very common in canines. Hepatic injuries cause serious health issues which may result in death of animals. Liver is a highly vascularized organ and chances of bleeding are very high in hepatic surgeries. Bleeding during surgery is a life threatening issue. Despite all recent developments in surgical techniques during liver surgery, blood loss is still one of the main causes for postoperative morbidity and mortality [1].

Hemostasis is the key to successful surgery which benefits the patient, the surgical team and the health care facility. The selection of correct hemostatic agent and the time of its application need understanding of the mechanism of action, efficacy and harmful effects of various hemostatic agents [2].

Approximately half of deaths due to trauma are due to exsanguinations[3]. Bleeding occur in traffic accidents remains one among the most causes of traffic accident deaths. To save the life by stopping or minimizing blood loss until advanced medical care is the main target of healthcare team[4]. Hemorrhage can occur after trauma which can leads to death. It is also the major cause of operating room mortality among patients who undergo liver surgery [5].After the establishment of safe airway and ensuring the proper oxygenation and ventilation, the best priority in the handling of the traumapatient is to control hemorrhage. As a result of patients could bleed from multiple sites at the same time[6].

Uncontrolled intra-operative bleeding is a serious complication that can impede both the surgeon's ability to complete the procedure and the patient's ability to recover. Blood that remains intra-abdominally after surgery not only increases the extent and severity of postoperative intestinal adhesions, but also serves as a source of intra-abdominal infections. Inadequate hemostasis increases operative time, recovery, and length of hospital stay, and constitutes a significant economic burden. Conventional methods used for obtaining hemostasis at continuously bleeding sites include repetitive direct cauterization, placement of sutures or clips, and prolonged direct compression, but these methods can impair tissue healing and recovery through the generation of tissue damage, burn, and necrosis. Conventional methods might also be useless or inefficient in areas that are hard to access or in organs and tissues located nearby that can be easily damaged (e.g. nerves or the respiratory tract). Hence, the development of more effective hemostatic agents is critical in order to keep surgical costs down and provide the best safety for patients [7]. 


\section{Materials and Methods}

\section{Dog Selection}

12 healthy mongrel dogs of either sex weighing from twelve to $15 \mathrm{~kg}$ were arranged from suburbs of Lahore city and maintained in the experimental kennels of Department of Clinical Medicine and Surgery of University of Veterinary and Animal Sciences, Lahore.

\section{Experimental Design}

Deworming and vaccination of the dogs along with complete clinical examination was done, and prior to initiation of study their temperature, heart rate, respiratory rate, capillary refill time, and general body condition was thoroughly checked. The dogs were divided in three groups $\mathrm{A}, \mathrm{B}$ and $\mathrm{C}$. In all groups a $3 \mathrm{~cm}$ long and $1 \mathrm{~cm}$ deep incision was given in left lateral lobe of liver. In group A, Surgical Gauzes were used to stop bleeding. In group B, Surgicel Snow was used to stop bleeding. In group C, Surgicel Fibrillar was used to stop bleeding. Prior to the surgery all dogs were kept off feed for 12 hours. In all groups, Atropine Sulphate was administered subcutaneously at the dose rate of $0.04 \mathrm{mg} / \mathrm{kg}$ as a pre anaesthetic; 15 minutes prior to the surgery. Groups A, B and C were generally anaesthetized using 5\% Ketamine (inj. Ketasole 50mg/ml) and $2 \%$ xylazine (inj. Xylaz $20 \mathrm{mg} / \mathrm{ml}$ ) combination intravenously at the dose rate of $5.5 \mathrm{mg} / \mathrm{kg}$ and $1.1 \mathrm{mg} / \mathrm{kg}$ respectively [8].

A ventral midline abdominal incision was given starting from xyphoid cartilage up to umbilicus. The length of incision was large enough to permit adequate exposure of the liver. The liver was lying over the stomach and caudal to diaphragm. The left lateral lobe was identified and lifted out through laparotomy incision [9]. A $3 \mathrm{~cm}$ long, $1 \mathrm{~cm}$ deep incision was given on left lateral lobe of liver and control the bleeding with Surgical Gauze, Surgicel Snow and Surgicel Fibrillar.

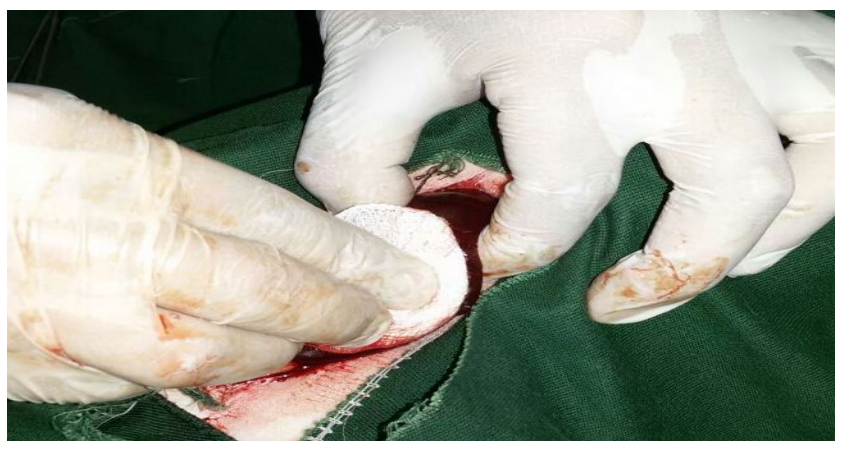

Figure1. Surgical Gauze used on Liver

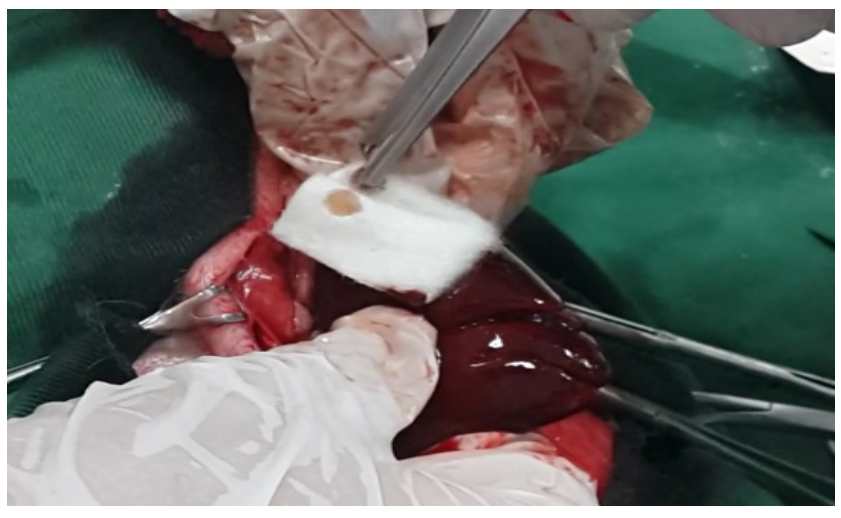

Figure 2. Surgicel Snow used on Liver

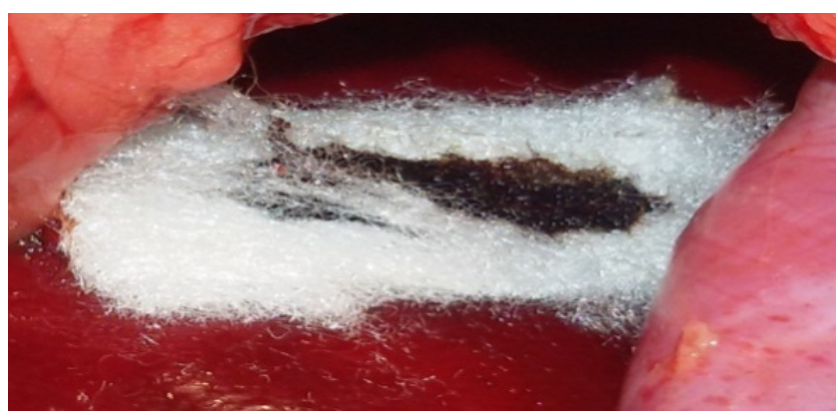

Figure 3. Surgicel Fibrillar used on Liver

Just in closure, the ventral midline abdominal incision was closed in three layers. Linea Alba was apposed by simple interrupted using the absorbable suture material (chromic catgut 2/0-Ethicon). Subcutaneous tissue was closed by using same suture material in simple interrupted fashion. The skin incision was closed by using non-absorbable (Mersilk silk suture 2/0-Ethicon) suture material in simple interrupted fashion.

The following parameters include Hemostasis time, LFT, CBC, TPR, and Ultrasonography were evaluated.

The data were analyzed by various techniques of One Way ANOVA with significance $\mathrm{P}<0.05$ by using Statistical Package for Social Science (SPSS) version 15.0.

\section{Results}

Hemostasis Time Period of Group A, B and C, treated with Surgical Gauze, Surgicel Snow and Surgicel Fibrillar was determined. Results are express in Mean $\pm \mathrm{SD},(n=4)$.

\begin{tabular}{|c|c|c|}
\hline Surgical Methods & Hemostasis time (sec) & P-Value \\
\hline Surgical Gauze & $201.00 \pm 5.34^{* * *}$ & \multirow{2}{*}{.000} \\
\cline { 1 - 2 } Surgicel Snow & $31.50 \pm 3.10^{*}$ & \\
\cline { 1 - 2 } Surgicel Fibrillar & $44.50 \pm 2.08^{* *}$ & \\
\hline
\end{tabular}

Figure 4. LSD test showed *minimum hemostasis time **higher $* * *$ maximum hemostasis time 


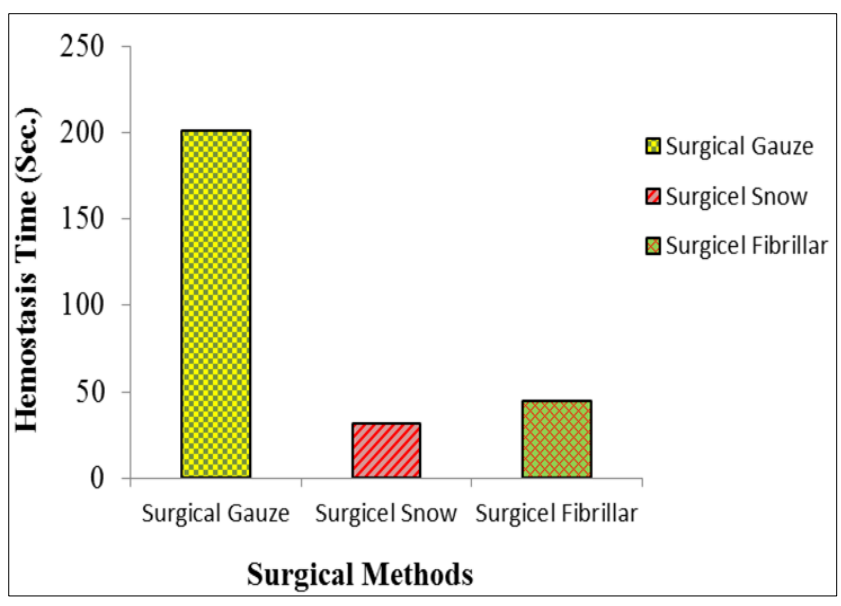

Figure 5. Mean Hemostasis Time of Group A, B and C, treated with Surgical Gauze, Surgicel Snow and Surgicel Fibrillar respectively
Graphical representation of all three treatment show minimum and maximum hemostasis time. Dogs treated with Surgicel Snow shows minimum hemostasis time followed by dogs of group C and group A. To evaluate the Liver Function Test of 12 dogs $(n=4)$ treated with Surgical Gauze, Surgicel Snow and Surgicel Fibrillar. Total 36 samples of blood were collected before surgery, after surgery, at 7 day and at 14 day to perform Alanine Amino-transferase (ALT), Aspartate Amino-transferase (AST), and Alkaline Phosphatase (ALP) tests.

For evaluation of ALT, AST and ALP test, blood samples were compared within all three $\mathrm{A}, \mathrm{B}$ and $\mathrm{C}$ groups by applying One-way ANOVA having level of significance $(\mathrm{P}<0.05)$ to compare any change among the groups

\begin{tabular}{|c|c|c|c|c|c|c|c|c|}
\hline \multirow{2}{*}{ Surgical Methods } & \multicolumn{2}{|r|}{ BS } & \multicolumn{2}{|r|}{ AS } & \multicolumn{2}{|r|}{7 DAS } & \multicolumn{2}{|r|}{14 DAS } \\
\hline & Mean \pm SD & P-Value $(P<0.05)$ & Mean \pm SD & P-Value $(P<0.05)$ & Mean \pm SD & P-Value $(\mathrm{P}<0.05)$ & Mean \pm SD & P-Value $(\mathrm{P}<0.05)$ \\
\hline Surgical Gauze & $38.00 \pm 7.88$ & \multirow{3}{*}{.944} & $81.75 \pm 5.12$ & \multirow{3}{*}{.003} & $59.00 \pm 3.74$ & \multirow{3}{*}{.015} & $45.6 \pm 5.80$ & \multirow{3}{*}{.275} \\
\hline Surgicel Snow & $39.25 \pm 7.90$ & & $66.75 \pm 4.86$ & & $47.76 \pm 5.38$ & & $39.6 \pm 5.00$ & \\
\hline Surgicel Fibrillar & $37.75 \pm 2.99$ & & $70.50 \pm 3.41$ & & $49.00 \pm 4.70$ & & $43.6 \pm 4.04$ & \\
\hline
\end{tabular}

Figure 6. Mean ALT U/L values Before Surgery, After Surgery, 7 days after Surgery and at 14 days after Surgery.

Mean values of ALT tests of Group A, B and C dogs ( $\mathrm{n}=4)$ after surgery and at 7 days after Surgery showed significant results while Before Surgery and at 14 days after Surgery showed non-significant results.

\begin{tabular}{|c|c|c|c|c|c|c|c|c|}
\hline \multirow{2}{*}{ Surgical Methods } & \multicolumn{2}{|r|}{ BS } & \multicolumn{2}{|r|}{ AS } & \multicolumn{2}{|c|}{7 DAS } & \multicolumn{2}{|c|}{14 DAS } \\
\hline & Mean \pm SD & P-Value $(\mathrm{P}<0.05)$ & Mean \pm SD & P-Value $(\mathrm{P}<0.05)$ & Mean \pm SD & P-Value $(\mathrm{P}<0.05)$ & Mean \pm SD & P-Value $(P<0.05)$ \\
\hline Surgical Gauze & $37.6 \pm 3.10$ & \multirow{3}{*}{.738} & $85.25 \pm 6.94$ & \multirow{3}{*}{.078} & $42.25 \pm 5.00$ & \multirow{3}{*}{.312} & $40.50 \pm 4.6$ & \multirow{3}{*}{.088} \\
\hline Surgicel Snow & $37.25 \pm 3.30$ & & $72.6 \pm 5.07$ & & $37.8 \pm 2.50$ & & $34.00 \pm 3.6$ & \\
\hline Surgicel Fibrillar & $35.25 \pm 6.13$ & & $78.00 \pm 8.29$ & & $40.8 \pm 4.03$ & & $37.25 \pm 2.6$ & \\
\hline
\end{tabular}

Figure 7. Mean AST U/L values Before Surgery, After Surgery, 7 days after Surgery and at 14 days after Surgery.

Mean values of AST tests of Group A, B and C dogs ( $n=4)$, P-Value showed non-significant results. 


\begin{tabular}{|c|c|c|c|c|c|c|c|c|}
\hline \multirow{2}{*}{ Surgical Methods } & \multicolumn{2}{|c|}{ BS } & \multicolumn{2}{|c|}{ AS } & \multicolumn{2}{|c|}{7 DAS } & \multicolumn{2}{|c|}{14 DAS } \\
\hline & Mean \pm SD & P-Value $(\mathrm{P}<0.05)$ & Mean \pm SD & P-Value $(P<0.05)$ & Mean \pm SD & P-Value $(P<0.05)$ & Mean \pm SD & P-Value $(\mathrm{P}<0.05)$ \\
\hline Surgical Gauze & $73.00 \pm 5.48$ & \multirow{3}{*}{.693} & $121.26 \pm 6.86$ & \multirow{3}{*}{.028} & $86.00 \pm 10.10$ & \multirow{3}{*}{.302} & $77.80 \pm 6.19$ & \multirow{3}{*}{.197} \\
\hline Surgicel Snow & $72.80 \pm 6.40$ & & $108.80 \pm 4.64$ & & $76.50 \pm 9.11$ & & $69.50 \pm 6.40$ & \\
\hline Surgicel Fibrillar & $69.25 \pm 8.20$ & & $113.00 \pm 4.54$ & & $77.80 \pm 6.94$ & & $73.00 \pm 5.16$ & \\
\hline
\end{tabular}

Figure 8. Mean ALP U/L values Before Surgery, After Surgery, 7 days after Surgery and at 14 days after Surgery.

Mean values of ALP tests of Group A, B and C dogs ( $\mathrm{n}=4)$ showed significant results only after surgery. While the P-value Before Surgery, At 7 Day and at 14 Days after Surgery showed non-significant results.

\begin{tabular}{|c|c|c|c|c|c|c|c|c|c|c|c|c|}
\hline \multirow{3}{*}{ Surgical Methods } & \multicolumn{3}{|c|}{ RBC's $* 10^{6} / \mu \mathrm{L}$} & \multicolumn{3}{|c|}{ WBC's $* 10^{3} / \mu \mathrm{L}$} & \multicolumn{3}{|c|}{ Platelets $* 10^{3} / \mu \mathrm{L}$} & \multicolumn{3}{|c|}{ Hemoglobin g/dL } \\
\hline & \multicolumn{2}{|c|}{ Mean \pm SD } & \multirow{2}{*}{ P-Value $(\mathrm{P}<0.05)$} & \multicolumn{2}{|c|}{ Mean \pm SD } & \multirow{2}{*}{ P-Value $(P<0.05)$} & \multicolumn{2}{|c|}{ Mean \pm SD } & \multirow{2}{*}{ P-Value $(P<0.05)$} & \multicolumn{2}{|c|}{ Mean \pm SD } & \multirow{2}{*}{ P-Value $(\mathrm{P}<0.05)$} \\
\hline & BS & AS 24 Hours & & BS & AS 24 Hours & & BS & AS 24Hours & & BS & AS 24 Hours & \\
\hline Surgical Gauze & $6.13 \pm .36$ & $5.36 \pm .40$ & .081 & $14.21 \pm 1.24$ & $18.81 \pm 1.42$ & .279 & $396.00 \pm 55.61$ & $516.50 \pm 35.81$ & .398 & $13.02 \pm .63$ & $10.96 \pm .44$ & .042 \\
\hline Surgicel Snow & $6.01 \pm .34$ & $5.33 \pm .29$ & .027 & $14.81 \pm 1.69$ & $19.91 \pm 1.46$ & .146 & $407.26 \pm 32.10$ & $471.00 \pm 44.51$ & .027 & $13.02 \pm .80$ & $11.78 \pm .63$ & .041 \\
\hline Surgicel Fibrillar & $6.02 \pm .26$ & $5.30 \pm .28$ & .152 & $14.49 \pm 1.49$ & $18.60 \pm .82$ & .141 & $421.00 \pm 25.47$ & $487.00 \pm 22.08$ & .411 & $13.32 \pm .83$ & $11.82 \pm .64$ & .047 \\
\hline
\end{tabular}

Figure 9. Mean values of RBC's, WBC's, Platelets and Hemoglobin before Surgery and 24 hours After Surgery.

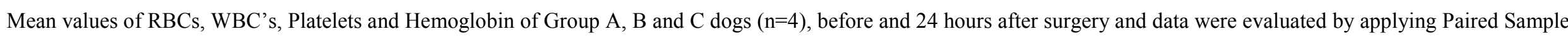

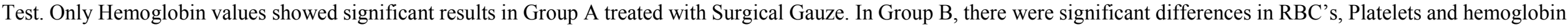
values before and 24 hours after surgery, while in Group C only Hemoglobin values showed significant differences.

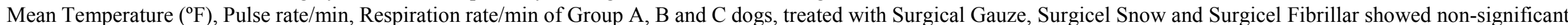
results.

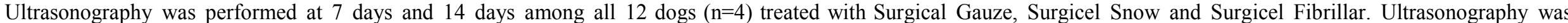

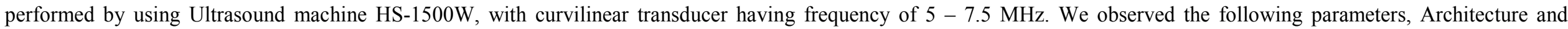
echogenicity, Free blood in abdomen, Hepatomegaly, Acute hepatitis. 


\begin{tabular}{|c|c|c|c|}
\hline Observations & Group A & Group B & Group C \\
\hline Architecture and echogenicity & $\begin{array}{c}\text { Hypoechoic } \\
\text { Grey colour } \\
\text { Sharpe edges } \\
\text { Normal texture }\end{array}$ & $\begin{array}{c}\text { Hypoechoic } \\
\text { Grey colour } \\
\text { Sharpe edges } \\
\text { Normal texture }\end{array}$ & $\begin{array}{c}\text { Hypoechoic, } \\
\text { Grey colour } \\
\text { Sharpe edges } \\
\text { Normal texture }\end{array}$ \\
\hline Free blood in abdomen & No & No & No \\
\hline Acute hepatitis & No & No & No \\
\hline Hepatomegaly & No & No & No \\
\hline
\end{tabular}

Figure 10. Ultrasonography of Liver at 7 days \& at 14 days after Surgery

We observed that there is no change on liver surface after applying three surgical methods. All three groups showed similar results.

\section{Discussion}

Oxidized regenerated cellulose (ORC) used as an absorbable hemostat since World War II[10]. Surgicel is a sterile bio absorbable thrombogenic agent used adjunctively in surgical procedures to assist in the control of capillary, venous, and small arterial hemorrhage when ligation or other conventional methods of control are impractical or ineffective[11]. Surgicel use in hepatobiliary surgery is where bleeding from small hepatic vessels may be challenging to control [12]. Oxidized regenerated cellulose used to control bleeding in open and endoscopic procedures, including laparoscopic partial nephrectomy [13] laparoscopic cholecystectomy [14] liver and spleen trauma [15]. Oxidized regenerated cellulose may be used for endoscopic procedures, and it may be cut to size to conform to the bleeding site[16]. Davidson and colleagues compared original ORC with autologous fibrin sealant in a swine model of partial hepatectomy and found that ORC and fibrin sealant both significantly reduced bleeding volume and hemostasis time compared to untreated controls [17].

A study was conducted to compare the effect of different hemostatic agents on bone healing in dogs, the authors, suggested that surgicel (oxidized cellulose) was a better hemostatic agent for bone healing compared to microfibrillar collagen and gel foam and contraindicated the use of bone wax[18]. Other research was performed to check the efficacy of Surgicel that proved effective for the reduction of bleeding after operations such as partial nephrectomy and presacral surgery [19].

The level of change of AST, ALT was high immediately after performing the hepatic operation in patients. These observations coincided with the study reported by Ahmad et al. [20]. Seven days following the hepatic surgery, both enzymes ALT and AST returned to normal value in patients. The findings are in agreement with the observations of Tan et al. [21]. In the following project LFT, ALP level was measured in which ALP level increased in the transplanted homologus dog liver that showed ALP level increase was the sign of fluctuation in the function of liver [22].

In a research there was a significant decrease in Red blood cell values in patients who were treated with surgery for acute cholecystitis [23]. A study was established to observe an increase of WBC of more than $20.000 / \mu 1$ early after OLT orthopedic liver transplantation was associated with patients in which liver was transplanted [24]. Platelets showed fluctuations after liver resection in hepatocellular carcinoma case [25].

An Ultrasonographic study was designed to check the febrile formation in the wounded liver resulted in liver dysfunction after laparotomy in rats that showed differences in normal liver after laparotomy in rats [26]. A project was formed to check the liver volume measurement has been reported in dogs with CPSS using radiography, ultrasonography, and CT [27].

\section{Conclusions}

On the basis of findings of this study: it was concluded Surgicel Snow is better hemostat than Surgical Fibrillar. It is the best hemostat with minimum blood loss and least effects on liver function.

\section{REFERENCES}

[1] Berrevoet, F. and B. de Hemptinne (2007). "Use of topical hemostatic agents during liver resection." Digestive Surgery 24(4): 288-293.

[2] Nooh, N., W. A. Abdullah, et al. (2014). "The effects of surgicel and bone wax hemostatic agents on bone healing: An experimental study." Indian journal of orthopaedics 48(3): 319.

[3] Bilgili, H., A. Kosar, et al. (2009). "Hemostatic efficacy of Ankaferd Blood Stopper ${ }^{\circledR}$ in a swine bleeding model." Medical Principles and Practice 18(3): 165-169.

[4] Nouri, S. and M. R. Sharif (2014). "Efficacy and safety of ferric chloride in controlling hepatic bleeding; an animal model study." Hepatitis monthly 14(6).

[5] Tokgöz, H., K. Karakaya, et al. (2010). "Protective value of a folkloric medicinal plant extract against mortality and hemorrhage in a life-threatening renal trauma model." 
Urology 75(6): 1515. e1519-1515. e1514.

[6] Plaisier, B. R. (2001). Surgical perspectives to control bleeding in trauma. Seminars in Anesthesia, Perioperative Medicine and Pain, Elsevier.

[7] Schuhmacher, C., J. Pratschke, et al. (2015). "Safety and effectiveness of a synthetic hemostatic patch for intraoperative soft tissue bleeding." Medical devices (Auckland, NZ) 8: 167.

[8] Franco, L. G., M. C. S. Fioravanti, et al. (2009). "Assessment of serum enzymatic markers of cardiomyocytes injury in female dogs submitted to ketamine $\mathrm{S}(+)$, atropin and xylazine association." Acta cirúrgica brasileira 24(1): 36-42.

[9] Slatter, D. H. (2003). Textbook of small animal surgery, Elsevier Health Sciences.

[10] Hutchinson, R. W., K. George, et al. (2013). "Hemostatic efficacy and tissue reaction of oxidized regenerated cellulose hemostats." Cellulose 20(1): 537-545.

[11] Insert, P. (2005). "Surgicel, Surgicel Fibrillar, and Surgicel Nu-Knit." Johnson \& Johnson.

[12] Oto, A., E. M. Remer, et al. (1999). "MR characteristics of oxidized cellulose (Surgicel). American journal of roentgenology 172(6): 1481-1484.

[13] Breda, A., S. V. Stepanian, et al. (2007). "Use of haemostatic agents and glues during laparoscopic partial nephrectomy: a multi-institutional survey from the United States and Europe of 1347 cases." European urology 52(3): 798-803.

[14] Rastogi, V. and V. Dy (2002). "Control of port-site bleeding from smaller incisions after laparoscopic cholecystectomy surgery: a new, innovative, and easier technique." Surgical Laparoscopy Endoscopy \& Percutaneous Techniques 12(4): 224-226.

[15] Theuer, C. P. and D. K. Imagawa (1999). "Use of knitted oxidized cellulose (Nu-knit) for the definitive packing of grade III liver fracture." Injury 30(2): 137-140.

[16] Gabay, M. (2006). "Absorbable hemostatic agents." American journal of health-system pharmacy 63(13): 1244-1253.

[17] Davidson, B., S. Burnett, et al. (2000). "Experimental study of a novel fibrin sealant for achieving haemostasis following partial hepatectomy." British Journal of Surgery 87(6):
$790-795$

[18] Finn, M. D., S. R. Schow, et al. (1992). "Osseous regeneration in the presence of four common hemostatic agents." Journal of oral and maxillofacial surgery 50(6): 608-612.Nooh, N., W. A.Abdullah, et al. (2014). "The effects of surgicel and bone wax hemostatic agents on bone healing: An experimental study." Indian journal of orthopaedics 48(3): 319.

[19] Abou-Elela, A., A. Morsy, et al. (2009). "Use of oxidized cellulose hemostats (Surgicel ${ }^{\mathrm{TM}}$ ) to support parenchymal closure and achieve hemostasis following partial nephrectomy." Surgical technology international 18: 75-79.

[20] Ahmad, N. Z. (2011). "Routine testing of liver function before and after elective laparoscopic cholecystectomy: is it necessary?" JSLS: Journal of the Society of Laparoendoscopic Surgeons 15(1): 65.

[21] Tan, M., F.-F. Xu, et al. (2003). "Changes in the level of serum liver enzymes after laparoscopic surgery." World journal of gastroenterology 9(2): 364-367.

[22] Starzl, T. E. and H. A. Kaupp (1961). "Studies on the rejection of the transplanted homologous dog liver." Surgery, gynecology \& obstetrics 112: 135.

[23] Yazıc1, P., U. Demir, et al. (2015). "What is the effect of treatment modality on red blood cell distribution width in patients with acute cholecystitis?" Turkish Journal of Surgery/Ulusal cerrahi dergisi 31(1): 1 .

[24] Helfritz, F., F. Lehner, et al. (2015). "Perioperative White Blood Cell Count as a Marker for Patient and Graft Survival after Orthotopic Liver Transplantation." J Hepatol Gastroint Dis 1(106): 2.

[25] Riediger, C., J. Bachmann, et al. (2013). "Low postoperative platelet count is associated with negative outcome after liver resection for hepatocellular carcinoma. "Hepato-gastroenter ology 61(133): 1313-1320.

[26] Dias, J., B. Paredes, et al. (2008). "An ultrasound and histomorphological analysis of experimental liver cirrhosis in rats." Brazilian Journal of Medical and Biological Research 41(11): 992-999.

[27] Washizu, M., M. katagi, et al. (2004). "An evaluation of radiographic hepatic size in dogs with portosystemic shunt." Journal of veterinary medical science 66(8): 977-9. 